\title{
Acute pain medicine in anesthesiology
} André P. Boezaart ${ }^{1,2 *}$, Anastacia P. Munro ${ }^{2}$ and Patrick J. Tighe ${ }^{2}$

Addresses: ${ }^{1}$ Department of Orthopaedic Surgery, University of Florida College of Medicine, 1600 SW Archer Road, PO Box 100254 , Gainesville, FL 32610, USA; ${ }^{2}$ Department of Anesthesiology, Division of Acute and Perioperative Pain Medicine, University of Florida College of Medicine, 1600 SW Archer Road, PO Box 100254, Gainesville, FL 32610, USA

*Corresponding author: André P. Boezaart (aboezaart@anest.ufl.edu)

Fl000Prime Reports 2013, 5:54 (doi:10.12703/P5-54)

This is an open-access article distributed under the terms of the Creative Commons Attribution-Non Commercial License (http://creativecommons.org/licenses/by-nc/3.0/legalcode), which permits unrestricted use, distribution, and reproduction in any medium, provided the original work is properly cited. You may not use this work for commercial purposes.

The electronic version of this article is the complete one and can be found at: http://fl000.com/prime/reports/m/5/54

\begin{abstract}
The American Academy of Pain Medicine and the American Society for Regional Anesthesia have recently focused on the evolving practice of acute pain medicine. There is increasing recognition that the scope and practice of acute pain therapies must extend beyond the subacute pain phase to include pre-pain and pre-intervention risk stratification, resident and fellow education in regional anesthesia and multimodal analgesia, as well as a deeper understanding of the pathophysiologic mechanisms that are integral to the variability observed among individual responses to nociception. Acute pain medicine is also being established as a vital component of successful systems-level acute pain management programs, inpatient cost containment, and patient satisfaction scores. In this review, we discuss the evolution and practice of acute pain medicine and we aim to facilitate further discussion on the evolution and advancement of this field as a subspecialty of anesthesiology.
\end{abstract}

\section{Introduction}

As acute pain begins to gain recognition as a disease process unto itself, we must pay attention not only to its immediate treatment but also to the underlying pathophysiology and reduction of its incidence and complications. Because of the complex nature of acute pain, there is a need for a subspecialty of anesthesiology that is devoted to the research, prevention, and adequate treatment of this condition. The field of anesthesiology is expanding its scope to conquer the complex landscape of acute pain in a society increasingly inclined toward surgical solutions. Furthermore, the subspecialty of acute pain medicine (APM) is well poised to extend its scope to the challenging management of acute and chronic pain, and palliative care and cancer treatment by potentially altering the immune response to surgery, and, by extension, to cancer growth and proliferation.

\section{History}

The concept of an acute pain service (APS) as an anesthesiology-based postoperative pain management service was introduced by Ready and colleagues in
1988 [1], with practice guidelines established first in Australia [2], followed by the United Kingdom [3], and then by the United States [4] in the early 1990s. Following these efforts, in 2001, the Joint Commission on Accreditation of Health Care Organizations put forth standards for the pain management of hospitalized patients [5]. Currently, APSs are evolving into services led by anesthesiologists seeking to manage pain in the pre-, intra-, and postoperative phases. This evolution is commensurate with the expanded capabilities and greater success rates of modern continuous regional anesthetic techniques as well as with the continually mounting evidence on the role of multimodal analgesics. In this context, the term "regional anesthesia" (RA) is inadequate, as it does not accurately describe the discipline or subspecialty of APM, which evaluates and treats surgical and nonsurgical pain in a dynamic and comprehensive way. RA focuses only on the interventional techniques offered by the discipline, which is akin to the way invasive hemodynamic monitoring, for example, does not accurately represent or describe the discipline of critical pain medicine. APM, in our view, more accurately describes a practice focused on acute pain 
and on surgical and nonsurgical, as well as on interventional and noninterventional, modalities.

\section{Evolving role of acute pain medicine}

Postoperative pain management services evolve into broader hospital-based acute pain programs when they begin to assist in the care of patients with acute pain outside the perioperative environment. This requires that APM teams work in close contact with the primary health care providers to fully optimize patient comfort and to manage and coordinate a patient's progression through the system.

In a 2002 editorial, Rawal offered six tenets essential to a modern APS service, which remain the core goals of the discipline today [6]. Rawal noted that the modern APM team must provide ongoing management, the assessment and treatment of pain as an underlying disease process, and administrative assistance through quality improvement and leadership; they must also serve as a source of education to the patients and staff. As noted by Rawal, because of the constrained financial resources supporting many pain programs, hospital-wide APSs must consult with and enlist additional experts to provide full and balanced care.

Chief among these additional experts are nurses facile with the management of acute pain. To this end, it is critical that APSs advocate for the training of specialized acute pain support staff who will have a critical role in standardizing pain assessment and treatment protocols, patient and staff education, and quality improvement efforts [7]. In fact, one of the main focus areas of APM evolution is the alignment of pain management practices such that administrative, medical, anesthetic, surgical, and nursing activities are implemented in a coordinated and complementary fashion.

To determine which APM activities offer the most value, APM services will need to rely on data stemming from local quality improvement efforts as well as from largescale structured research programs. The development of "Big Data" repositories of pain-related outcomes, such as from the Multicenter Perioperative Outcomes Group, the National Surgical Quality Improvement Program, and Pain-OUT, can help drive decision making for patientand systems-level challenges [8-10]. These repositories can also aid in improving the currently available metrics of pain by offering insights into the association between acute pain scores and long-term outcomes.

APM services will continue to evolve via consultation into physical and temporal environments external to the perioperative arena. For instance, the APM service may aid in the management of palliative care by offering additional analgesic modalities, including interventional procedures, to certain patients who may benefit [11]. The APM service may also serve as a transition and referral point for outpatient chronic pain interventions, a role that may become more vital in accountable care organization models of pain care. By establishing a service with 24-hour in-house capability that can augment pain assessments, pharmacological management, complementary therapies, and RA techniques, the APM can offer multiple evaluatediagnose-treat pain management cycles per day and extend their capabilities when the operating room is quiescent, thus permitting a more cost-effective implementation of advanced pain care in a manner similar to that currently offered in many hospitals for obstetric patients.

\section{Cost-to-benefit ratio of acute pain service}

One of the major difficulties in assessing the costeffectiveness of the APS lies in the difficulty of defining an APS. Current tools for evaluating costs, which lack sensitivity to pain outcomes and quantifiable functional outcomes, have an inherent inability to differentiate average effects from individual effects, and depend on a response across several alternatives to pain management [12]. Furthermore, there is a well-known lack of consensus regarding the standards for staffing, support, or objective outcome measurements for evaluating APS performance [12]. Although limited, current meta-analyses of APS teams have shown that cost savings are evident as measured by shorter intensive care unit (ICU) stays [13], shorter hospital length of stay [13], potential reduction of the incidence of chronic pain [13], and better survival outcomes for certain cancers [14] —all at the comparable cost of a daily patient-controlled analgesia (PCA) unit. In addition, a patient's assessment of satisfactory pain control is closely tied with his or her perception of the quality of a hospital and his or her willingness to recommend said hospital to others (Press Ganey score) [15]. In the United States, the recent linkage of HCAHPS (Hospital Care Quality Information from the Consumer Perspective) scores to hospital reimbursements by CMS (Centers for Medicare and Medicaid Services) further implicates poor pain management in fiscal losses for acute care hospitals [16].

\section{Complementary supplementation}

The current reliance on pharmacological modalities, specifically opioids, as the primary method of pain control has led to their misuse, to increased incidences of tolerance and addiction, as well as to increased adverse side effects such as hyperalgesia, pruritus, and postoperative nausea. A dedicated APS focuses on multimodal analgesia while minimizing the use of opioids and promotes alternative 
pain management strategies such as acupuncture [17], massage therapy $[18,19]$, transcutaneous electrical nerve stimulation [20,21], and even guided imagery [22]. The supportive evidence for these modalities has led to their acceptance by many institutions as part of the standard analgesic critical pathways.

\section{Environment and staffing}

To ensure a functional APM service, hospitals must provide a supportive physician and cultural work environment. These can be subdivided into administrative duties, rounding, and consulting and RA facilities.

The environment for administrative duties requires an established workspace in which providers can safely and securely store materials and patient data, gather such data for meetings and conferences, and collect data for quality improvement. Furthermore, resources are necessary to support multidisciplinary pain rounds, continuing education efforts, and the tracking of patient outcomes and adverse events, ensuring the continued growth of an APM service.

As rounding and consulting traditionally occur at a patient's bedside, the requirements for an efficient and successful service may include the provisioning of supplies for dressing reinforcement, local anesthetics, basic resuscitation equipment, nerve block equipment, and portable ultrasound equipment to assess and manage those patients for whom the current analgesic plan is inadequate. Rounding teams may wish to carry supplies for resuscitation in rare cases of hemodynamic instability, respiratory depression, or local anesthetic systemic toxicity. Having the capability to evaluate medical records, including pain histories and opioid prescription records from pharmacy and/or state databases, is also critical to ensure efficient and thorough evaluation of patient histories during consultations.

RA should take place in dedicated block rooms, although it should be noted that these rooms might also be used to initiate opioid or ketamine infusions and provide other noninterventional approaches in an accelerated fashion. Specialized block rooms offer convenience and increased efficiency of block placement because they can be staffed with a highly trained APM team and stocked with the necessary supplies to provide RA while offering methods for closely monitoring patients post-RA and assessing the efficacy of the block prior to surgical intervention. This has the potential to reduce risk and improve patient safety and satisfaction by quickly treating pain exacerbations. These dedicated block rooms may also provide an environment congruent with teaching RA and assessing quality control. In addition, dedicated block rooms serve to improve operating room turnover times and facilitate on-time, first-case starts by allowing nerve blocks to be done in parallel with operating room cases.

Because of the scheduling demands of most hospitals, nerve blocks are often performed in operating rooms prior to surgery. This practice puts unnecessary time pressure on practitioners, which invariably leads to failed blocks and the increased risk of complications. Using the operating room to perform nerve blocks is an undesirable, costineffective practice that should be discouraged: there is a lack of readily available materials and equipment, and the time of the operating room personnel could be managed more efficiently. Finally, as with the other operating room staff, surgeons are losing valuable time at the start of surgery as well while the block is being performed.

It is vital that time delays be avoided; if nerve blocks consistently impact surgery start times, these delays in the induction of anesthesia may cause surgeons to avoid RA as a pain management technique [23]. To that effect, dedicated block rooms can streamline patient flow and create an efficient system for the provision of interventional pain management techniques. Block room costs can be offset by using the space for post-anesthesia care unit or general preoperative evaluations, depending on time of day, surgical volume, and staffing. This crossutilization may also facilitate patient flow for the entire perioperative process.

\section{Fellowship programs: past and future}

Despite the rapidly growing field of perioperative and non-perioperative acute pain management, few existing fellowship programs are designed to adequately train physicians on the broad approach to APM. Current pain fellowship programs that function primarily in outpatient pain clinics are not commonly designed to provide around-the-clock hospital care nor allow one to remain current in the rapidly expanding field of acute pain treatment. Emerging acute pain fellowship programs are shifting focus to treating acute pain by embracing the continuity between acute and chronic pain. There is still a wide variability in the forms of training provided.

APM can be broadly divided into interventional analgesic techniques (RA) and acute pain management. The depth of knowledge and extensive procedural proficiency required to master RA necessitate that a significant portion of training and education be devoted to this area during fellowship training. Expanding on the 2010 guidelines for fellowship training [24], several changes were proposed that suggest a more holistic and multimodal approach must take place, including placing more emphasis on the APM physician as a consultant who is able to integrate 
multiple services; a leader able to lead multidisciplinary meetings; a competent clinician; an instructor of medical students, residents, fellows, and colleagues; and a competent "regional anesthesiologist". An argument can be made for partnering with chronic pain fellowship programs that could offer fellows in both disciplines opportunities to collaborate on difficult patients where the distinctions between acute and chronic pain may be blurred. Such an overlap would be thematically similar to that between neurology, physical medicine and rehabilitation, and the currently structured pain fellowships. Following the current Accreditation Council for Graduate Medical Education (ACGME) core competencies for pain medicine will allow for the more defined medical knowledge base necessary for practicing APM and will place emphasis on the multimodal treatment of various types of pain. This can include a deeper understanding of pain neurophysiology (pain neurotransmitters, nerve fibers, and central and peripheral pain pathways) as well as common pain syndromes. Fellows should gain an understanding of the contribution of biopsychosocial factors that are often an integral part of a pain condition. Complementary, alternative medicine techniques that are increasingly used in APM should also be familiar to APM fellows. In addition, as RA evolves from an intraoperative tool to a comprehensive set of options for controlling postoperative pain, knowledge of the appropriate outpatient pain management options and ways to transition to such regimens will be invaluable to a future APM physician.

\section{Acute pain medicine team members}

To establish a competent APS, there must be a team managed by a comprehensively trained physician who will function as the cornerstone of that team. Midlevel health-care providers, pharmacists, technicians, and clerks play important roles in the modern APM team.

Attending physicians place and follow the nerve blocks, fulfill consulting duties, and lead the rounding team on once- or twice-daily ward rounds. The organization and behavior of such a team will vary according to institutional resources and norms but at the very least should include input from primary care teams, nursing teams, and APM physicians. This role should be distinct from that of placing nerve blocks, given the escalating consultative nature in many APM programs. In some institutions, the nerve block follow-up is relegated to a midlevel provider; however, we believe that the final responsibility for the safe and effective relief of acute pain remains with anesthesiologists, and thus the attending physician should be not only performing the block but also conducting the follow-up care. There are various examples where complications such as compartment syndrome have been prevented, or at least had their impact lessened, by the APS teams "demystifying" the effects of nerve blocks, especially continuous nerve blocks [25-27].

Pharmacists provide an important role in the safe provision of pharmacological therapy for acute pain. It is suggested that APM physicians maintain a presence on the hospital Pharmacy and Therapeutics Committees to gain an understanding of new therapies, to familiarize themselves with new evidence regarding pharmaceuticals, and to interact with other stakeholders while offering input toward applicable decisions.

Nurses are a vital and integral part of any APM team. The APM nurse is tasked with a multidimensional role that includes patient follow-up, including standardization of protocols and data collection for quality improvement; education of bedside nurses and patients; direct provision of complementary and alternative medicine therapies; and support of APM interventions. The APM nurse is also able to facilitate communication between physical and occupational therapists, social workers, psychologists, and other health-care professionals involved in a patient's care and to triage patients that fall outside the scope of the practice of APM and divert them elsewhere as appropriate.

Nurses who specialize in assisting with continuous and single-injection nerve blocks are professionals trained in preoperative assessment and sedation and they are able to assist with block placement and post-anesthesia and postblock placement care of the patient. They are also tasked with the sterile preparation of block placement equipment, the provisioning of preemptive sedation, and proper patient positioning and patient monitoring. This subspecialty requires a thorough understanding of the nature of nerve blocks used, familiarity with ultrasound and nerve stimulators, and the ability to recognize and treat block-related adverse events.

\section{The future of acute pain medicine}

Despite advances in acute pain management, a significant number of patients continue to report experiencing significant postsurgical pain. It must be emphasized that the provision for an APS would ensure the proper and timely management of acute pain with multimodal analgesia and interventional techniques; it would also propagate education and provide for evidence-based analgesic pathways in a standardized fashion. In embracing the continuum of acute pain in the surgical and nonsurgical arena and shifting focus to "preventative analgesia" (i.e. treating acute pain in all surgical phases in the hopes of preventing chronic pain and debilitation), future research can center on the patient as a whole and on 
identifying preoperative risk factors, intraoperative occurrences, and postoperative rehabilitation modalities that may decrease the incidence of acute pain and minimize its untoward effects on patient recovery. New developments into the field of improvement of cancer surgery outcomes through the use of RA to obliterate the surgical stress response are very exciting and promising $[14,28]$.

Current research has suffered from multiple design flaws, including lacking true placebo arms and failing to address how inconsistent measures of success are reported in the literature $[29,30]$. Study designs should include validation of patient satisfaction, hospital stay, and ability to perform physical therapy exercises, in addition to the currently accepted pain scores and amount of opioid usage. Measurements focusing on indices of mood, sleep, and level of stress can prove to be valued additions to APM quality assessment. In addition to broadening the view of acute pain in the form of pre-pain or pre-intervention risk stratification, researchers should elucidate the specificity for surgery type and condition as well. Future research must aim to establish condition- and procedure-specific analgesic regimens that would account for the temporal nature of pain and the unique nature of different conditions.

Given the explosion of research and knowledge on the complexities of acute pain, APM is poised to become a subspecialty akin to chronic pain and addiction medicine. As anesthesiology evolves into the field of perioperative medicine, physicians must evolve to understand acute pain as a disease process and not solely focus on the tools of RA. Collaboration among surgeons, primary care providers, midlevel health-care providers, and nurses will be essential in establishing APM not only as a viable option but as a necessity in any hospital setting.

\section{Abbreviations}

APM, acute pain medicine; APS, acute pain service; ICU, intensive care unit; PCU, patient-controlled analgesia; $\mathrm{RA}$, regional anesthesia.

\section{Disclosures}

Dr. Boezaart serves as a consultant for TeleFlex Medical (Limerick, PA, USA) and receives royalty payments from them. The other authors declare that they have no competing interests.

\section{Acknowledgments}

The authors would like to acknowledge that this article was inspired and influenced by the work of Drs. Justin Upp, Michael Kent, and Patrick Tighe [31]. They also thank Rebecca C. Astrom (Corey) for her help with the preparation of this manuscript.

\section{References}

I. Ready LB, Oden R, Chadwick HS, Benedetti C, Rooke GA, Caplan R, Wild LM: Development of an anesthesiology-based postoperative pain management service. Anesthesiology 1988, 68: 100-6.

2. Australian Government National Health and Medical Research Council: Emergency Care Acute Pain Management Manual; 1988 (updated 1999, 2005). [http://www.nhmrc.gov.au/_files_nhmrc/ publications/attachments/cp|35_emergency_acute_pain_management_ manual.pdf]

3. Royal College of Surgeons of England, The College of Anesthetists: Pain after surgery; 1990 [http://www.rcoa.ac.uk/system/files/FPMPain-After-Surgery.pdf]

4. American Pain Society: Principles of Analgesic Use in the Treatment of Acute and Chronic Cancer Pain. Clin Pharm 1990, 9:60I-12.

5. American Society of Anesthesiologists Task Force on Acute Pain Management: Practice guidelines for acute pain management in the perioperative setting: An updated report by the American Society of Anesthesiologists Task Force on Acute Pain Management. Anesthesiology 2004, 100:I573-8I.

\section{FlOOOPrime}

RECOMMENDED

6. Rawal N: Acute pain services revisited - Good from far, far from good? Reg Anesth Pain Med 2002, 27:I I7-2I.

\section{FIOOOPRime
RECOMMENDED}

7. Rawal N: 10 years of acute pain services - achievements and challenges. Reg Anesth Pain Med 1999, 24:68-73.

FlOOOPrime

RECOMMENDED

8. Multicenter Perioperative Outcomes Group. [http://mpog.med. umich.edu/about-mpog]

9. American College of Surgeons: National Surgical Quality Improvement Program. [http://site.acsnsqip.org/]

10. Zaslansky R, Chapman CR, Rothaug J, Backstrom R, Brill S, Davidson E, Elessi K, Fletcher D, Fodor L, Karanja E, Konrad C, Kopf A, Leykin Y, Lipman A, Puig M, Rawal N, Schug S, Ullrich K, Volk T, Meissner W: Feasibility of international data collection and feedback on post-operative pain data: Proof of concept. Eur J Pain 2012, 16:430-8.

FlOOOPrime
RECOMMENDED

II. Esch AT, Esch A, Knorr JL, Boezaart AP: Long-term ambulatory continuous nerve blocks for terminally ill patients: $A$ case series. Pain Med 2010, I I:I299-1302.

12. Sun E, Dexter F, Macario A: Can an acute pain service be costeffective? Anesth Analg 2010, I I I:84 I-4.

\section{FlOOOPrime}

13. Lee A, Chan S, Chen PP, Gin T, Lau AS: Economic evaluations of acute pain service programs: A systematic review. Clin J Pain 2007, 23:726-33.

\section{FlOOOPrime \\ RECOMMENDED}

14. Chen WK, Miao $\mathrm{CH}$ : The effect of anesthetic technique on survival in human cancers: $A$ meta-analysis of retrospective and prospective studies. PLoS One 2013, 8:e56540.

\section{FlOOOPrime}

RECOMMENDED

15. Press Ganey (ed.): Hospital pulse report; 2009. [http://www. pressganey.com/Documents_secure/Pulse\%20Reports/Hospital_Pulse_ Report_2009.pdf?viewFile] 
16. Aston G: Smart pain management makes good business sense. Hosp Health Netw 2012, 86:38-40, 49-50.

\section{FIOOOPrime}

17. Towler P, Molassiotis A, Brearley SG: What is the evidence for the use of acupuncture as an intervention for symptom management in cancer supportive and palliative care: An integrative overview of reviews. Support Care Cancer 2013, [Epub ahead of print].

\section{FIOOOPRIM
RECOMMENDED}

18. Carinci AJ, Pathak R, Young M, Christo PJ: Complementary and alternative treatments for chronic pain. Curr Pain Headache Rep 2013, I7:316.

19. Running A, Seright T: Integrative oncology: Managing cancer pain with complementary and alternative therapies. Curr Pain Headache Rep 2012, 16:325-31.

20. Nizard J, Lefaucheur JP, Helbert M, de Chauvigny E, Nguyen JP: Non-invasive stimulation therapies for the treatment of refractory pain. Discovery Med 20I2, I4:2I-3I.

21. Sbruzzi G, Silveira SA, Silva DV, Coronel CC, Plentz RD: Transcutaneous electrical nerve stimulation after thoracic surgery: Systematic review and meta-analysis of I I randomized trials. Rev Bras Cir Cardiovasc 2012, 27:75-87.

\section{FlOOOPrime

RECOMMENDED

22. Posadzki P, Lewandowski W, Terry R, Ernst E, Stearns A: Guided imagery for non-musculoskeletal pain: A systematic review of randomized clinical trials. 20I2, 44:95-104

\section{FlOOOPrime
RECOMMENDED}

23. Masursky D, Dexter F, McCartney CJ, Isaacson SA, Nussmeier NA: Predicting orthopedic surgeons' preferences for peripheral nerve blocks for their patients. Anesth Analg 2008, 106:56 I-7.
24. Hargett MJ, Beckman JD, Liguori GA, Neal JM: Education Committee in the Department of Anesthesiology at Hospital for Special Surgery: Guidelines for regional anesthesia fellowship training. Reg Anesth Pain Med 2005, 30:218-25.

25. Cometa MA, Esch AT, Boezaart AP: Did continuous femoral and sciatic nerve block obscure the diagnosis or delay the treatment of acute lower leg compartment syndrome? A case report. Pain Med 20I I, I 2:823-8.

26. Walker BJ, Noonan KJ, Bosenberg AT: Evolving compartment syndrome not masked by a continuous peripheral nerve block: Evidence-based case management. Reg Anesth Pain Med 20I2, 37:393-7.

27. Aguirre JA, Gresch D, Popovici A, Bernhard J, Borgeat A: Case scenario: Compartment syndrome of the forearm in a patient with an infraclavicular catheter: Breakthrough pain as indicator. Anesthesiology 2013, I I 8: I I 98-205.

28. Christopherson R, James KE, Tableman M, Marshall P, Johnson FE: Long-term survival after colon cancer surgery: a variation associated with choice of anesthesia. Anesth Analg 2008, I07: 325-32.

\section{FlOOOPrime} RECOMMENDED

29. Andreae $\mathrm{MH}$, Andreae DA: Regional anesthesia to prevent chronic pain after surgery: A Cochrane systematic review and meta-analysis. $\mathrm{Br} J$ Anaesth 2013, [Epub ahead of print].

\section{FlOOOPrime}

RECOMMENDED

30. Andreae $\mathrm{MH}$, Andreae DA: Local anesthetics and regional anesthesia for preventing chronic pain after surgery. Cochrane Database Syst Rev 2012, 10:CD007105.

\section{FlOOOPrime}

\section{RECOMMENDED}

31. UpP J, Kent M, Tighe PJ: The evolution and practice of acute pain medicine. Pain Medicine 2013, | 4:|24-|44. 